\title{
Factors Affecting the Decision Making of HPV Vaccination Uptake Among Female Youth in Klang Valley (Influencing Factors): A Qualitative Study
}

\author{
Siti Nur Farhana Harun ${ }^{1}$, Mazrura Sahani ${ }^{2} \&$ Mohammad Zabri Johari $^{1}$ \\ ${ }^{1}$ Institute for Health Behavioural Research, Ministry of Health Malaysia, Malaysia \\ ${ }^{2}$ Faculty of Health Sciences, Universiti Kebangsaan Malaysia, Malaysia \\ Correspondence: Siti Nur Farhana Harun, Institute for Health Behavioural Research, Ministry of Health Malaysia, \\ Jalan Rumah Sakit Bangsar, 59000 Kuala Lumpur, Malaysia. Tel: 60-3-2082-1400. E-mail: farhana@moh.gov.my
}

\author{
Received: October 1, 2018 Accepted: November 12, 2018 Online Published: December 13, 2018 \\ doi:10.5539/gjhs.v11n1p36 URL: https://doi.org/10.5539/gjhs.v11n1p36
}

\begin{abstract}
Introduction: Cervical cancer is estimated to affect 500000 women each year globally, whereby $80 \%$ of the cases are in developing nations. Almost all cervical cancer cases were attributed to Human Papilloma Virus (HPV) infection.
\end{abstract}

Aim: To identify factors influencing the decision-making of HPV vaccination uptake as prevention for cervical cancer among female youth in the Klang Valley

Methods: This study used in-depth interview; purposive sampling and snowball sampling method. The questionnaire was based on the Health Belief Model, which consist of perceived susceptibility, severity, benefit, barrier and cues to action. NVivo 7 software was used to process, transcribe and analyse the data from interview sessions.

Result: This study found that the key driving factors that encouraged female youth to get vaccinated were due to the role of family members and friends, concerns on contracting HPV related illness, free/discounted priced vaccination, recommendation from health care personnel, government's policy, and benefit (believe in the effectiveness of vaccination). Meanwhile, deterring factors which prevented the uptake of HPV vaccination were lack of knowledge and awareness, costs, healthcare provider and services, time constraint and perceived not at risk.

Conclusion: Factors leading to the uptake of the HPV vaccine should be seen in a transparent manner to ensure the success of the HPV vaccination program in this country.

Keywords: HPV vaccine, cervical cancer, qualitative

\section{Introduction}

Cervical cancer is estimated to affect 500000 women each year globally, whereby $80 \%$ of the cases are in developing nations (Cutts et al., 2007). Studies have shown that HPV 16 is the most oncogenic, accounting for almost half of all cervical cancers, and HPV 16 and 18 together account for approximately $70 \%$ of cervical cancers. HPV 6 and 11 are the most common strains associated with genital warts and are responsible for approximately 90\% of these lesions (Braaten \& Laufer, 2008). Genital HPV types are divided into high and low-risk types, according to the oncogenic potential. Molecular and epidemiologic studies have solidified the association between high-risk HPV types (especially HPV-16 and HPV-18) and cervical squamous cell carcinoma. HPV infection is often transient and self-limiting but uncommonly, the infection persists and progress to high grade lesions and cancer. In addition to persistent high-risk HPV infection, other viral factors such as high viral loads, HPV variants, infections with multiple high-risk HPV types and genetic predisposition contribute to the development of cervical cancer (Gomez \& Santoz, 2007). In cervical cancer, conventional Papanicolaou smear (Pap smear) remains the main screening method, as it has been proven to reduce incidences by $43 \%$ and decreases its mortality by $46 \%$ (Cohn \& Herzog, 2001). The sensitivity of Pap smear has a wide range from as low as $30 \%$ to as high as $87 \%$ indicating that many cases could be missed by this screening test. The specificity is $86-100 \%$ indicating that there are false positive diagnoses made (Hayati, 2003). Prevention via vaccination could be a more effective method 
compared to just screening. Vaccination is more relevant in developing countries as a comprehensive screening program are not feasible due to limited resources (Ministry of Health Malaysia, 2011). This study was conducted to explore factors influencing the decision-making process of HPV vaccination uptake as prevention for cervical cancer amongst female youth in Klang Valley. Furthermore, this study also attempts to ascertain the level of knowledge, awareness and understanding perceptions of female youth regarding cervical cancer and HPV vaccination, factors influencing the uptake of HPV vaccine and the deterring factor for HPV vaccine uptake.

\subsection{Prevention Strategies}

The Malaysian government approved HPV vaccination program with three doses of HPV vaccine freely given to all 13- year-old girls from public or private schools on 21st February 2008; and the budget was approved on 19th August 2009. World Health Organization (WHO) recommended that routine HPV vaccination be included in National Immunization programs (Zaridah, 2014). According to the Annual Report of Malaysia Ministry of Health (2012), immunisation coverage achievement of a complete three dosage of HPV vaccine among 13-year-old girls in the year 2012 was $99.2 \%$.

The Human Papilloma Virus (HPV) Quadrivalent Vaccine was approved by the Malaysian Drug Authority on 20th October 2006 while the Bivalent Vaccine was approved in 2007 (Rushdan, 2008). The vaccines are given in three doses and each injection are given within six months (month 0,2,6) (LPPKN, 2012). The optimal target age for prophylactic vaccination is pre-pubertal girls before coitarche (age 9-14 years). Catch-up vaccination involves slightly older women (15-18 years) (Kyrgiou \& Shafi, 2008).

\section{Material and Methods}

A qualitative study was conducted between 1st September till 30th January 2016 in Klang Valley, Kuala Lumpur. Randomization selection of study sites were carried out and three youth centers were selected. Participants were selected based on characteristics such as age group, gender, race, vaccinated and not vaccinated to ensure diversification of participants ranging through ethnicity, age, and personal background. Snowball sampling was used to ensure continuity of recruitment with suitable characteristics for this study. No sample size was determined since the study used a qualitative method whereby the important aspect in determining sample size for qualitative research is the saturation of information. The method of repetitions from the answers by the participants for all interview questions are the pre-determined criteria to stop data collection. This study used a sample selection and data analysis through an ongoing process until at a point where no new information or information obtained from the current interview was overlaid with information obtained earlier. Another method used by qualitative researchers to determine whether information collected has reached the saturation point is when researchers found that the participants began providing the same idea over and over again and no new information that can be shared.

Interview questions were developed from the specific objectives of the study; questions were derived from the Health Belief Model that consisted of five main constructs; perceived susceptibility (participants' susceptibility to HPV infection, risk to contract HPV infection, participants behavior to avoid the risk, participants' family health history), perceived severity (the severity expected if infected with HPV, participants' confidence in HPV vaccine to prevent cervical cancer), cues to action (motivation to find out the factors that encourage participants to take HPV vaccine), perceived benefit (benefits of getting HPV vaccine, the importance of HPV vaccine to the participants), and perceived barrier (emphasized to identify the obstacles faced by participants that prevented participants in getting the HPV vaccine) and perceived threat (to find out if threat is the driving factor to get HPV vaccine) (Rosenstock et al., 1994). For the first pre-test, a list of semi-structured interview questions was constructed and validated by feedback from two subject matter experts on HPV and health promotion and behavior. It was piloted to two participants selected randomly at a youth center in Klang Valley to ensure that the interview questions can be understood and to identify the gaps found in the interview questions.

\subsection{Data Collection, Management, and Analysis}

Participants were interviewed by a trained interviewer. The interviews were carried out in either in Malay or English language depending on participants' preference, lasting approximately for 30 to 45 minutes every session. After the pilot study, improvisation were made and the researcher continued interviewing 15 other female participants in Klang Valley after reaching repetitions of answers for all questions. Data obtained from the interview sessions were transcribed verbatim and captured on the NVivo 7 software. Accuracy concerning the substance of the interview, the meanings and perceptions created and shared during a conversation was maintained through verbatim transcription. This was also done to not only capture the meaning and perception or the recorded interviews and focus group discussions, but also the context in which these were created. Discussion with the technical expert was carried out to avoid bias in this study through intercoder reliability when recognizing themes. 
An Environmental Health expert was invited to give input on technical judgment on public health and a Senior Health Promotion Expert gave a technical opinion based on health promotion perspectives. Researcher organized key themes using deductive analysis guided by HBM framework (Rosenstock et al., 1994).

\subsection{Ethical Issues}

Ethical approval was obtained from the Medical Research and Ethics Committee, Ministry of Health Malaysia.

\section{Results}

\subsection{Socio-Demographic}

Basic ethnical data revealed that eight participants were Malays, two Chinese and five Indians. All participants were between 18 and 24 years old. Sociodemographic characteristic is depicted in Table 1.

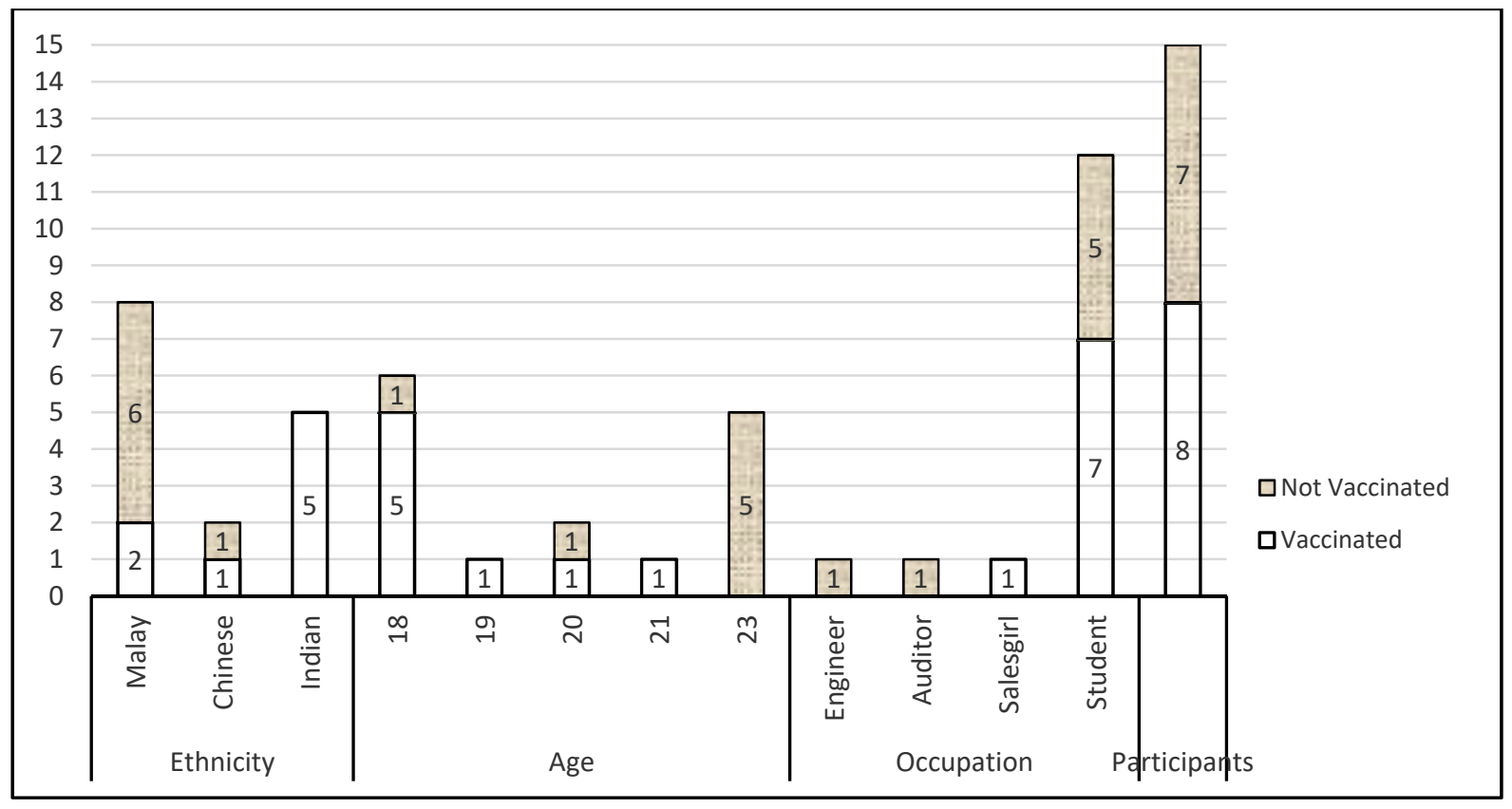

Table 1. Socio-demographic

Code:

$\mathrm{R}=$ Participant's code number.

M, C, I= Malay, Chinese, Indian (ethnicity).

$\mathrm{V}=$ Vaccinated.

$\mathrm{NV}=$ Not vaccinated.

\subsection{Level of Knowledge and Understanding}

\subsubsection{Understanding of Cervical Cancer (Causal Factors)}

Understanding aspects of cervical cancer were evaluated from the participants' understanding of the occurrence cause of cervical cancer. The majority of participants were able to provide answers in fundamental factors causing cervical cancer. Amongst the answers that are often given by the participants to answer the question on factors that cause cervical cancer are sexually promiscuous, heredity or genetics and hygiene.

"Do you mind to explain what do you understand about cervical cancer?"

"Cervical cancer occurs near the cervix due to; one, genetics, two, sexual promiscuity and It may also be due to the environment. Environment factor such as cleanliness at female's private part was not kept clean can lead to cervical cancer "(R14, M, V). 


\subsubsection{Understanding of HPV Vaccine}

Based on the interviews with participants, it was found that almost all participants have a low understanding on HPV vaccine. Very few participants knew that the HPV vaccine is to prevent cervical cancer while other participants admitted that they do not know and do not get enough information about the HPV vaccine.

"Can you explain what do you understand about HPV vaccination?"

"I think it is to prevent from getting cervical cancer." (R12,I,V)

"To be honest I do not know what HPV vaccine is. I have heard of it but I do not know what it does "(R1, M, $\mathrm{NV})$.

\subsection{Awareness}

\subsubsection{Awareness on the Importance of HPV Vaccination}

Through an analysis of in-depth interview, majority gave similar answers when asked about the importance of HPV vaccination. The most common answer is that HPV vaccination is known as a preventive measure for cervical cancer. Most participants also explained the important function of HPV vaccination is to reduce a woman's risk of contracting the human papilloma virus (HPV).

"In your opinion, what is the importance of HPV vaccination that you know?"

"The importance of the vaccine is to prevent us from getting infected by cervical cancer. Another thing is that we do not know what will happen and it is a benefit because we are still young. So better take it now as it won't be effective when we're old. Fear it would not be effective " (R14, M, V).

"The importance is a step taken to prevent and not regretting once you got cancer. (R11,I,V)

\subsubsection{Awareness of the HPV Vaccination Regimen}

A bigger number of participants knew the total number of dosages for HPV vaccination to be taken. However, a smaller number were unaware or unsure. Two participants did not answer the questions raised and only one gave an accurate answer about the HPV injection regime as stated below. Majority of the participants were able to state the number of injections needed which was three (3) times.

"Are you aware of HPV vaccine regimen? Do you realize how many doses do you need?"

"I have done it. The first one, and then you take the second one after two months, and then the third one after 6 months" (R10, C, V).

"I am not sure. But I think few doses." (R15,M,NV)

\section{Factors Influencing the Uptake of HPV Vaccine}

This study found the key driving factors for female youths to get HPV vaccination was due to the role of family members and friends, concerns on contracting HPV related illness, free/ discounted priced vaccination, recommendations from health care personnel, government's policy and benefit (believe in the effectiveness of the vaccine). Factors preventing female youth getting HPV vaccination were lack of knowledge and awareness, cost, health care provider and services, time constraint and perception of "not at risk".

\subsection{Role of Family Members and Friends}

Overall, the role of family members and friends were considered to be the biggest driving factor for HPV vaccine uptake, both to participants who had been vaccinated or unvaccinated. Almost all participants felt that parents and friends played a big role in influencing to get HPV vaccination. Five participants implied that parents were the strongest influencers to ensure their acceptance for HPV vaccination and seven participants stated that friends and the people around them influenced them the most in their decision to get HPV vaccination.

"What motivates you to get the vaccination?"

"My parents and also friends from my school. My friends, they said it is important to get the vaccination and also from the nurse who gave the briefing about the HPV.”(R6, I, V).

"I don't want to take it at first, but my mom made me. She said "you have to take it, compulsory. It's good for health". (R12,I,V)

\subsection{Concerns on Contracting HPV Related Illness}

Concerns on contracting HPV related illness is the second important factor encouraging decision making of participants to opt for HPV vaccination in this study. During the in-depth interviews, more than half of the 
participants mentioned they were concerned about the dangers of HPV related illness and the threat of it was a strong motivating factor for them to be vaccinated or to have an intention for opting to vaccinate. According to the participants, the threat of the deadly HPV related illness triggered their action in getting vaccinated.

"Do you think you have the risk of getting infected?

"Because all women are likely of contracting the illness. So I get frightened by it. We are also women, hence there's definitely a chance of contracting it". (R7, M, NV)

"For me, the threat is big enough for me to think to take the vaccine". (R14,M,NV)

\subsection{Free/Discounted Priced Vaccination}

This study found that price reduction for vaccination through promotions was one of the driving factors for HPV vaccine uptake. About half of the participants mentioned by giving vaccination at a promotional price or free of charge will encourage female youths to opt for HPV vaccination. A total of six participants admitted opting for vaccination because it was free and one of the participants indicated that it is worthwhile to get vaccinated at a promotional price and the rest communally agreed if the vaccination was provided at a lower price.

"What do you think of the cost?"

"Maybe if you go to a private clinic, one injection cost you RM200 or RM100 plus. If it's not for the promotion, it's much more expensive, people will not go. I think it's worthy to get it when they do the promotion." $(\mathrm{R} 10, \mathrm{C}, \mathrm{V})$.

"If the vaccine is given for free, I can find time to go." (R7,M,NV)

"It's expensive. But then the government gave it for free." (R12,I,V)

\subsection{Recommendation from Healthcare Personnel}

The analysis also revealed recommendations from health personnel were seen to have an impact in influencing the decision to opt for HPV vaccination among female youths. Although only few of the participants gave such feedback, researchers felt this component to be vital. Participants explained, recommendations from doctors, advice from nurses and other health personnel such as Health Education Officers provided awareness so that they felt it was important to get HPV vaccination to prevent cervical cancer.

"What motivates you to get the vaccination?"

"The nurse once told this vaccine is to prevent cervical cancer, therefore she encouraged us to take." $(\mathrm{R} 8, \mathrm{M}, \mathrm{V})$

"If it is suggested by the doctor to take the shot, I might". (R5,I,V)

\subsection{Government's Policy}

This study found that five participants who had been vaccinated while they were in school. Majority mentioned it was considered compulsory for all female students. Government policy mandated 13-year-old school girls to receive HPV vaccination was a stimulus for female youths to be vaccinated. However, there were also participants who went on their own initiative to get vaccinated as they were not eligible for the free vaccination at the school since it wasn't available during their time.

"I got it at school. Compulsory. If it is not compulsory, I won't go and get it (vaccine)" (R5,I,V)

"I just get it because it is compulsory". (R3,I,V)

\subsection{Benefit (Believe in the Effectiveness of Vaccination)}

Participants mentioned they believed in the effectiveness of vaccination and its benefit in terms of health. Participants explained they felt safe and worry-free after being vaccinated.

"It's like when you get yourself vaccinated indirectly you put yourself under control, you might not get the infection. When you do random sexual intercourse, it is like you are putting yourself in danger. So this vaccine can help." (R5,I,V)

"For me, it is to prevent us from cancer. So I do not have to worry about my future. HPV vaccine will protect me from cancer. That's good". (R8, M, V)

\subsection{Factors Hindering the Uptake of HPV Vaccination}

\subsubsection{Lack of Knowledge and Awareness}

The biggest contributor to the deterring factors of getting vaccinated was due to the lack of knowledge and 
awareness on HPV vaccination. The majority acknowledged less exposure to HPV vaccination, lack of awareness on the importance of HPV vaccination and inadequate information resulting in participants feeling that HPV infection is less serious and not dangerous. Hence, the uptake of the HPV vaccine was not perceived as a priority by the participants.

“Because I'm not aware of its importance. It looks like it (HPV vaccine) is not important at all.”(R13, M, NV)

"Perhaps my lack of knowledge regarding that matter, I don't think it is serious." (R15,M,NV)

\subsubsection{Costs}

Cost was seen as a major deterrent in influencing the decision-making of getting HPV vaccination. Majority expressed cost as the biggest hurdle for them to get vaccinated. HPV vaccination costs RM180 for one dose and it is required to take three doses; this was considered too expensive, especially for students.

"For me, as I don't come from a well-off family. So if the HPV vaccine costs and money, may not opt for it." (R9, M, V)

"Because if three injections, I have to pay RM540, that is too much." (R10,C,V)

\subsubsection{Healthcare Provider and Services}

Participants indicated that healthcare providers and timing of healthcare service contributed to the hindrance in getting vaccinated. Other than being afraid to see the doctor, they also felt that doctors' approach when interacting with patients on the topic of the reproductive system and other sensitive topics (sexual health topic) tend to be negative, hence they will try to avoid seeing the doctors. On top of this, being unaware of operating hours and places that offer vaccination were commonly mentioned as barriers.

"Mostly because they are feeling shy to open up about this topic and mostly in our country the specialist are male doctors so they do not get the exposure to go and talk about this kind of topic and the same goes to breast cancer." (R11, I, V)

"I don't know the place that provides HPV vaccine service." (R14, M, NV)

"It's how the doctor approach, or maybe I am afraid of doctors. So that might be a barrier for me." (R1,M,NV)

\subsubsection{Time Constraint}

Due to intense weekly working schedule, participants were reluctant to use their weekend time to get vaccinated but preferred to spend the available time with their family. Furthermore, the existing time limitation for obtaining vaccination (HPV vaccination in Malaysia has a certain/specific schedule time; not available daily) reduces the likelihood of participants getting the vaccination. If they felt they truly needed the vaccination, they must apply for annual leave; which they perceived as a waste.

"Time contributes to the hindering factors. Maybe we are students, we have class daily and other activities on the weekend." (R14,M,NV)

"Hectic working schedule and limited free time are indeed a barrier. Besides, long range of regimen (6 months) might result in participant to forget the schedule." (R8,M,V)

\subsubsection{Not at Risk}

Another barrier commonly mentioned was participants were not interested in getting HPV vaccines due to the perception of "not being at risk". Participants explained they did not engage in sexual intercourse and always practiced a healthy lifestyle. Therefore, the risk for them being infected was perceived as low and felt it was pointless to take the vaccine if they were not at risk.

"If we don't have sexual intercourse means we have no possibility of being infected." (R13,M,NV)

"I think it is impossible to happen to me. If I did not do things that would put me at risk, it would be impossible to get the infection. Yes, that's what I mean." (R1,M,NV)

\section{Discussion}

\subsection{Family Members and Friends}

Family members and friends serve as a catalyst for change in one's behavior. This study found that the majority of participants felt that parents and friends influence them to get vaccinated and give a large weight to the decision-making process. If parents and friends responded positively to the HPV vaccine, thus higher potential for female youth to get vaccinated. These findings support previous studies that showed the urge to act, for instance, the suggestions from friends, family or recommendations from doctors as a strong predictor for the uptake of HPV 
vaccine (Bowyer et al. 2014).

\subsection{Concerns of Contracting the Illness}

Concerns of contracting the illness is one of the strong factors for behaviour change. Based on the findings, participants expressed their concerns on the dangers of illness and threats of it which; giving a strong motivating factor for them to take the vaccination. The threat of deadly illness triggered their behavior of getting vaccinated and expected high susceptibility in individuals who have health awareness can help them receive positive change when it comes to health. Individuals who have high awareness on health do not look at the obstacles as the problems, in fact, will try their best to see the positive side of it if it is beneficial to health. This finding is in congruence with other study conducted by Manhart et al. (2011) and Young et al. (2010) in which stated the assumption of getting cervical cancer is high may be the most important psychosocial factor that causes women intending to have such vaccine. The perception of the severity of the high cervical cancer increases the intention of participants to get the HPV vaccine. Women will take action to get vaccinated because they realized the severity of the illness that can cause death (Oscarrson et al., 2012).

\subsection{Free/Discounted Priced Vaccination}

HPV vaccination given at a discounted price or free could increase the expected benefits for young people to obtain $\mathrm{HPV}$ vaccination. Provision of subsidies can also be seen to overcome the barriers of getting vaccinated. One of the most powerful obstacles identified in the decision to opt for vaccination is the cost of the HPV vaccine (Conroy et al., 2009). This is because the regimen for the vaccine requires women to have 3 doses to complete and they are costly for them to take this vaccine. Each dose as indicated by the LPPKN could cost RM180 and 3 doses are approximately RM540 (Khor, 2018). With such cost, not all women can afford the vaccination especially for parents that have more than one daughter. The cost to vaccinate all their daughters would be greater. Therefore, the subsidies by concerned parties are needed to increase the uptake of the HPV vaccine.

\subsection{Benefit (Believe in Effectiveness of HPV Vaccination)}

The finding suggested perceived benefits and perceived effectiveness of HPV vaccination was associated with the acceptance of HPV vaccination. This finding has similarity with Chikandiwa (2013) which stated perceived vaccine effectiveness was associated with greater willingness to be vaccinated. Participants believed the benefit of getting themselves vaccinated, it will prevent them from contracting the illness.

\subsection{Recommendation from Healthcare Personnel}

In this study, participants stated the importance of recommendations from health personnel. According to them, it encourages their acceptance of HPV vaccination. This confirms the finding of the previous study by Chikandiwa (2013), which stated a doctor's recommendation for the vaccine, would result in a potential $84 \%$ vaccine uptake, while a recommendation from a spouse, friend or family member would lead to an approximate $60 \%$ uptake. A physician's recommendation of a vaccine has been identified as an important catalyst for vaccine acceptance. A number of studies have reported that higher acceptability of the vaccine was found in subjects who thought that their doctors would recommend it.

\subsection{Government's Policy}

Policy-level interventions have been shown to be effective in increasing public health benefits. Government policies and mandates may result in improved HPV vaccination coverage and reduced disease burden, and alternative policies that improve unhindered access to HPV vaccination may allow success as well (Brandt 2016). Therefore, the Malaysian government took steps by approving HPV vaccination program with three doses of HPV vaccine freely given to all 13-year-old girls from public or private schools beginning 21st February 2008 (Zaridah 2014); Government policy mandated 13 -year-old schoolgirls to receive HPV vaccines had become an enforcing factor for female youths to receive vaccines. By making it compulsory, this had strengthened HPV vaccination as the primary prevention of cervical cancer.

\section{Barriers in the Uptake of HPV Vaccine}

\subsection{Lack of Knowledge and Awareness}

The biggest contributor to the deterring factors of getting vaccinated is due to the lack of knowledge and awareness of the HPV vaccine. Majority of the participants stated that less exposure to HPV vaccine, lack of awareness about the importance of HPV vaccination and inadequate information resulting the negative perception from participants; HPV infection is less serious and not dangerous. Hence, the uptake of the HPV vaccine is not given a priority by the participants. In addition, from the analysis of the study found the main factor that prevented female youth who have not been vaccinated to get vaccinated are due to the lack of knowledge and awareness. The finding is in line 
with research finding of Wong \& Sam (2010) in which found a lack of knowledge and awareness regarding HPV vaccine could lead to a perception of less risk in contracting the illness and hence affect the decision-making in the uptake of HPV vaccine.

\subsection{Costs}

Undeniably, cost is a vital factor that affects the decision-making in the uptake of the HPV vaccine. There are many studies that have been conducted to prove this statement. Results showed that the majority of participants stated that cost is the biggest hurdle for them to get vaccinated. HPV vaccination costs RM180 for one injection (Khor, 2018) and is considered too expensive, especially for the students. The studies in the Western countries show similarities whereby the high cost for the injection has been identified as a critical barrier in getting vaccinated. The fee of US\$360 is beyond the means of most women. Although many women show positive intention to get vaccinated, the high cost has become the main obstacle to behavioral prevention. Subsidies from the government is required to reduce the financial burden and indirectly to increase the uptake of the HPV vaccine in Asian countries (Hyunjin, 2011).

\subsection{Healthcare Provider and Services}

Results showed that participants felt that the service is deemed to be a barrier for them to get vaccinated. According to the participants, other than being afraid to see the doctor, they also felt the doctors' approach when interacting with patients on the topic of the reproductive system tend to be negative. In line with the findings of Rambout et al. (2014), social norms and values of a culture are important. As for Asia in particular, issues such as sex is considered a taboo and prohibited to be discussed in public. The findings of the qualitative study found that some health personnel try to avoid conversations and discussions about HPV vaccine for fear it was not appropriate to the culture.

\subsection{Time Constraint}

Time constraint is an important barrier that will affect the decision to take HPV vaccine as explained by the participants. The findings of this study is consistent with the findings from Hopkin (2013) which indicate that among the causes of negative acceptance of women is time constraints and they do not consider HPV vaccination as a priority. Support from employers is indispensable because the time of HPV vaccination services limited to working days requires the sacrifice of a female employee to seek leave from the employers if they wish to get HPV vaccination on working days. This inconvenience will lead to the refusal. This finding is in congruence with other studies that reveal busy schedules and other priorities such as family, work, and school were also barriers from getting HPV vaccine and completing the 3 doses (Mills, 2013).

However, a small number of participants in the study stated that time is not a barrier because health care to prevent cervical cancer is more important so they should be wise in managing their time to get the HPV vaccine. This opinion is parallel to Forster et al. (2012) where researchers found that the main factor of women aged 16 to 18 intending to get vaccination was because of the efficacy of the vaccine in protecting them from cervical cancer and HPV infection. This shows that young women who are aware of the benefits of taking HPV vaccine for good health are the main reason they get HPV vaccine.

\subsection{Not at Risk}

Various misconceptions have arisen since HPV vaccine is introduced. The majority of participants have an understanding that the HPV vaccine is only for women who has engaged with multiple sexual partners. The analysis found that participants were not interested to get themselves vaccinated for the reason they did not perceive themselves to be at risk. Participants explained they have taken precautions to protect them by practicing safe sex or even some of them were never engaged in sexual intercourse, therefore, the risk for them being infected is low. This misrepresentation should be rectified so that the women out there will have the proper knowledge about this issue. Cancer is a disease that is difficult to anticipate and everyone is at risk for this lethal illness. Thus, cancer prevention should be addressed by all the same. Discoveries reported by Wong (2008) Many young women felt that they did not need the vaccine or would prefer to wait because they were not sexually active; this highlights the failure to educate women of the importance of vaccination before exposure to HPV. Young women must be made aware that the vaccine is most effective if administered before initiation of sexual activity (Goldie et al., 2004). Belief that only sexually active teenagers need to be vaccinated can cause parents to reject or delay HPV vaccination. Parents may underestimate their child's sexual experiences by saying they are unlikely to have such early sex and this has the potential for their children to miss the primary prevention opportunities. 


\section{Conclusion}

The results of this study can be used to change the community's perception towards HPV vaccination and place HPV vaccination as the primary prevention measure for cervical cancer. Most previous studies only looked at certain scopes; for instance the costs for HPV vaccination or factors hindering uptake of HPV vaccine. This study gives a significant value as it considers a wider scope in terms of knowledge, understanding, factors influencing uptake of HPV vaccination and the deterring factor for HPV vaccine uptake. The awareness on HPV vaccine is extremely important to ensure the increased uptake of HPV vaccine among female youth. Women should be made aware on the risks of HPV infection and its relation to cervical cancer. Many women are unaware that HPV infection is among the largest contributor to cervical cancer. Factors leading to the uptake of HPV vaccine should be seen in a transparent manner to ensure the success of HPV vaccination program in this country. On another note, the factors hindering the uptake of HPV vaccine should be studied in depth to find an effective solution in facilitating women for getting vaccinated.

\section{Acknowledgments}

The authors would like to thank the Director General of Health, Malaysia for permission to publish this report. The authors would like to extend their appreciation to everybody involves in this study.

\section{Funding}

No funding sources.

\section{Competing Interests Statement}

The authors declare that there are no competing or potential conflicts of interest.

\section{References}

Bowyer, H. L., Forster, A. S., Marlow, L. A., \& Waller, J. (2014). Predicting human papillomavirus vaccination behaviour among adolescent girls in England: results from a prospective survey. Journal of Family Planning and Reproductive Health Care, 40(1), 14-22. https://doi.org/10.1136/jfprhc-2013-100583

Braaten, K. P., \& Laufer, M. R. (2008). Human papillomavirus (HPV), HPV-related disease, and the HPV vaccine. Reviews in obstetrics and gynecology, 1(1), 2.

Brandt, H. M., Pierce, J. Y., \& Crary, A. (2016). Increasing HPV vaccination through policy for public health

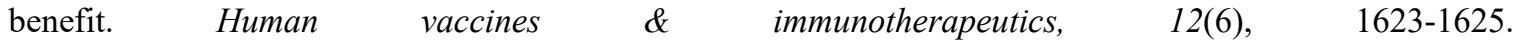
https://doi.org/10.1080/21645515.2015.1122145

Chikandiwa, A., \& van Wyk, B. E. (2013). Knowledge and awareness of human papillomavirus and intention with regard to human papillomavirus vaccine uptake by female tertiary students in the Eastern Cape province. Southern African Journal of Epidemiology and Infection, 28(4), 215-220. https://doi.org/10.1080/10158782.2013.11441553

Cohn D. E., \& Herzog T. J. (2001). New Innovations In Cervical Cancer Screening. Clin Obstet and Gynecol, 44(3), 538-549. https://doi.org/10.1097/00003081-200109000-00009

Cutts, F. T., Franceschi, S., Goldie, S., Castellsague, X., De Sanjose, S., Garnett, G., \& Markowitz, L. (2007). Human papillomavirus and HPV vaccines: a review. Bulletin of the World Health Organization, 85(9), 719-726. https://doi.org/10.2471/BLT.06.038414

Forsters, A. S. Marlow, I.A.V., Wardle, J., Stephenson, J., \& Waller, J. (2010). Understanding adolescent intention to have the HPV vaccine. Vaccine, 28, 1673-1676. https://doi.org/10.1016/j.vaccine.2009.11.082

Goldie, S. J., Kohli, M., Grima, D., Weinstein, M. C., Wright, T. C., Bosch, F. X., \& Franco, E. (2004). Projected clinical benefits and cost-effectiveness of a human papillomavirus $16 / 18$ vaccine. Journal of the National Cancer Institute, 96(8), 604-615. https://doi.org/10.1093/jnci/djh104

Gomez, D. T., \& Santos, J. L. (2007). Human papillomavirus infection and cervical cancer: pathogenesis and epidemiology. Communicating current research and educational topics and trends in applied microbiology, 1, 680-8.

Hayati, O. N. (2003). Cancer of the cervix-from bleak past to bright future; a review, with an emphasis on cancer of the cervix in Malaysia. The Malaysian journal of medical sciences: MJMS, 10(1), 13.

Hopkins, T. G., \& Wood, N. (2013). Female human papillomavirus (HPV) vaccination: global uptake and the impact of attitudes. Vaccine, 31(13), 1673-1679. https://doi.org/10.1016/j.vaccine.2013.01.028 
Khor, S. (2018, March 16). LPPKN Is Giving Out Free HPV Vaccinations For M'sian Women Born In 1991 To 1996 This Year. Retrieved August 7, 2018, from http://says.com/my/news/share-free-hpv-vaccinations-for-malaysian-women-born-in-1991-to-1996

Kyrgiou, M., \& Mahmood, I. S. (2009). HPV vaccine. Obstetric, gynecology and reproductive medicine, 19(1), 26-28. https://doi.org/10.1016/j.ogrm.2008.09.010

LPPKN. (2012). Info Pemvaksinan. Retrieved 22 September, 2015, from http://www.lppkn.gov.my/index.php/ms/perkhidmatan-kesihatan-reproduktif/99-pvhpv-human-papilloma-vi rus

Manhart, L. E., Burgess Hull, A. J., Fleming, C. B., Bailey, J. A., Haggerty, K. P., \& Catalano, R. F. (2011). HPV vaccination among a community sample of young adult women. Vaccine, 29, 5238-5244. https://doi.org/10.1016/j.vaccine.2011.05.024

Ministry of Health Malaysia. (2011). Health Technology Assessment Section Medical Development Division Ministry of Health Malaysia 008/2011.

Ministry of Health Malaysia. (2012). Annual Report Ministry of Health 2012. Kuala Lumpur: Ministry of Health Malaysia.

Mohd, R. (2008). Current status and future direction of cervical cancer prevention program in Malaysia. Retrieved November, 2015, from http://www.hopehandbook.org/

Oh, H. (2011). Knowledge about HPV, and the attitudes toward HPV vaccination among adult women in Asian countries: a literature review. Journal of Korean Oncology Nursing, 11(3), 171-178. https://doi.org/10.5388/jkon.2011.11.3.171

Rambout, L., Tashkandi, M., Hopkins, L., \& Tricco, A. C. (2014). Self-reported barriers and facilitators to preventive human papillomavirus vaccination among adolescent girls and young women: a systematic review. Preventive medicine, 58, 22-32. https://doi.org/10.1016/j.ypmed.2013.10.009

Rosentock, I. M. (1963). Public response to cancer screening and detection programs determinants of health behaviors. J. Chron. Dis., 16, 407-428. https://doi.org/10.1016/0021-9681(63)90117-6

Wong, L. P. (2008). Young multiethnic women's attitudes toward the HPV vaccine and HPV vaccination. International Journal of Gynecology \& Obstetrics, 103(2), 131-135. https://doi.org/10.1016/j.ijgo.2008.07.005

Wong, L. P., \& Sam, I. C. (2007). Current issues facing the introduction of human papillomavirus vaccine in Malaysia. Malaysian Family Physician: the Official Journal of the Academy of Family Physicians of Malaysia, 2(2), 47.

Young, A. M., Crosby, R. A., Jagger, K. S., Richardson, M. B., Kloha, R. A., \& Safarian, V. (2010). HPV vaccine acceptability among women in the Philippines. Asian Pac J Cancer Prev, 11(3), 1781-7.

Zaridah, S., \& UKM, M. (2014). A review of cervical cancer research in Malaysia. The Medical journal of Malaysia, 69, 33-41.

\section{Copyrights}

Copyright for this article is retained by the author(s), with first publication rights granted to the journal.

This is an open-access article distributed under the terms and conditions of the Creative Commons Attribution license (http://creativecommons.org/licenses/by/4.0/). 